\title{
Methyl Dynamics of a $\mathrm{Ca}^{2+-}$ Calmodulin-Peptide Complex from NMR/SRLS
}

\author{
Yury E. Shapiro ${ }^{1,}{ }^{*}$, Antonino Polimeno ${ }^{2}$, Jack H. Freed ${ }^{3}$, and Eva Meirovitch ${ }^{1},{ }^{*}$ \\ ${ }^{1}$ The Mina and Everard Goodman Faculty of Life Sciences, Bar-Ilan University, Ramat-Gan \\ 52900 Israel \\ ${ }^{2}$ Department of Physical Chemistry, University of Padua, 35131 Padua, Italy \\ ${ }^{3}$ Baker Laboratory of Chemistry and Chemical Biology, Cornell University, Ithaca, NY \\ 14853-1301, U.S.A.
}

\begin{abstract}
We developed the slowly relaxing local structure (SRLS) approach for analyzing NMR spin relaxation in proteins. SRLS accounts for dynamical coupling between the tumbling of the protein and the local motion of the probe, and for general tensorial properties. It is the generalization of the traditional model-free (MF) method, which does not account for mode-coupling and treats only simple tensiorial properties. SRLS is applied herein to ${ }^{2} \mathrm{H}$ relaxation of ${ }^{13} \mathrm{CDH}_{2}$ groups in the complex of $\mathrm{Ca}^{2+}$-calmodulin with the peptide smMLCKp. Literature data comprising ${ }^{2} \mathrm{H} T_{1}$ and $T_{2}$ acquired at 14.1 and $17.6 \mathrm{~T}$, and 288, 295, 308 and $320 \mathrm{~K}$, are used. We find that modecoupling is a small effect for methyl dynamics. On the other hand, general tensorial properties are important. In particular, it is important to allow for the asymmetry of the local spatial restrictions, which can be represented in SRLS by a rhombic local ordering tensor with components $S_{0}^{2}$ and $S_{2}^{2}$. Here, we find that $-0.2 \leq S_{0}^{2} \leq 0.5$ and $-0.4 \leq S_{2}^{2} \leq 0$. MF features a single "generalized" order parameter, $S$, confined to the $0-0.316$ range. The parameter $S$ is inaccurate, having absorbed unaccounted for effects, notably $S_{2}^{2} \neq 0$. We find that the methionine methyls (the other methyl types) reorient with rates of $8.6 \times 10^{9}-21.4 \times 10^{9}\left(0.67 \times 10^{9}-6.5 \times 10^{9}\right) 1 / \mathrm{s}$. The corresponding activation energies are $10(10-27) \mathrm{kJ} / \mathrm{mol}$. By contrast, MF yields inaccurate effective local motional correlation times, $\tau_{\mathrm{e}}$, with non-physical temperature-dependence. Thus, the problematic $S$-, and $\tau_{\mathrm{e}}$-based MF picture of methyl dynamics has been replaced with an insightful physical picture based on a local ordering tensor related to structural features, and a local diffusion tensor that yields accurate activation energies.
\end{abstract}

\section{Keywords}

slowly relaxing local structure (SRLS); methyl dynamics in proteins; ${ }^{2} \mathrm{H}$ spin relaxation in protein methyl groups

\section{Introduction}

NMR spin relaxation is a powerful method for elucidating protein dynamics. ${ }^{1-13}{ }^{15} \mathrm{~N}$ spin relaxation of the $\mathrm{N}-\mathrm{H}$ bond is typically used to study backbone dynamics, ${ }^{1-5,7-10}$ and ${ }^{2} \mathrm{H}$ spin relaxation of the ${ }^{13} \mathrm{CDH}_{2}$ methyl group, $5^{,}, 11^{-13}$ to study side-chain dynamics. We 
developed in recent years the slowly relaxing local structure (SRLS) approach ${ }^{14-} 16$ for analyzing NMR spin relaxation in proteins $17^{-} 19$. So far, SRLS has been implemented in the overdamped diffusion limit within the scope of a two-body coupled-rotator formalism.

SRLS accounts for dynamical coupling between the local motion of the NMR probe and the global motion of the protein, and allows for general properties of the second rank tensors involved. Here, we focus on methyl dynamics in the complex of $\mathrm{Ca}^{2+}-$ calmodulin with the peptide smMLCKp (GSARRKWQKTGHAVRAIGRLS), denoted $\mathrm{Ca}^{2+}-\mathrm{CaM}^{*} \mathrm{smMLCKp}$. Literature data comprising ${ }^{2} \mathrm{H} T_{1}$ and $T_{2}$ acquired at 14.1 and $17.6 \mathrm{~T}$, and 288, 295, 308 and $320 \mathrm{~K},{ }^{20}$ are analyzed with SRLS.

Methyl dynamics probed with ${ }^{13} \mathrm{CDH}_{2}$ (cf. ref 11) is inherently more challenging than backbone dynamics probed with ${ }^{15} \mathrm{~N}-{ }^{1} \mathrm{H} .{ }^{21}$ Both have been treated mostly with the modelfree (MF) approach. ${ }^{22-} 24$ While the local restrictions at the $\mathrm{N}-\mathrm{H}$ bond may be modeled as axial confinement around the equilibrium $\mathrm{N}-\mathrm{H}$ orientation, 22 this picture is not applicable to methyl motion. It was shown in early work that axial confinement around the $\mathrm{C}-\mathrm{CH}_{3}$ bond, accounted for straightforwardly within the scope of Woessner's model, 25 does not explain experimental data from proteins.26 Rather, non-axial restrictions on the motion of this bond must also be accounted for. ${ }^{26}$ Thus, although the local motion of the methyl group is typically much faster than the global tumbling (hence largely decoupled from it), ${ }^{6}, 27^{-} 30$ as assumed in MF, appropriate treatment of methyl dynamics requires accounting for general tensorial properties. MF cannot account for these properties.

Unlike the ${ }^{15} \mathrm{~N}$ nucleus in the ${ }^{15} \mathrm{~N}-{ }^{1} \mathrm{H}$ probe, the ${ }^{2} \mathrm{H}$ nucleus in the ${ }^{13} \mathrm{CDH}_{2}$ probe is not bound chemically to ${ }^{1} \mathrm{H}$. Hence, the important high-frequency values of the spectral density, supplied by heteronuclear NOEs, are not available. Only the spectral density values of

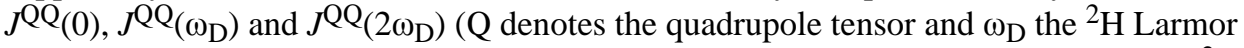
frequency) enter the expressions for the experimental relaxation parameters, typically ${ }^{2} \mathrm{H} T_{1}$ and $T_{2} \cdot{ }^{11}$ Finally, the $T_{2}$ values of the quadrupole ${ }^{2} \mathrm{H}$ nucleus are small, and often quite similar at different magnetic fields.

In SRLS, the local restrictions are represented by a (coupling/ordering) potential of mean force (POMT). The simplest representation of the non-axial local ordering scenario described above is through a rhombic POMT with the main local ordering axis, lying along the $\mathrm{C}-\mathrm{CDH}_{2}$ bond, i.e., tilted at $110.5^{\circ}$ from the (axial) quadrupolar frame (this value of the tetrahedral carbon angle corresponds to $r_{\mathrm{CH}}=r_{\mathrm{CD}}=1.115 \AA$, used in methyl HH-HC crosscorrelation studies27).

This model was suggested by us previously and applied to experimental data. ${ }^{28,29}$ For simplicity, we considered the local diffusion tensor, $\boldsymbol{R}^{\mathbf{L}}$, to be isotropic. In the present study we investigate the temperature dependence of the local motional rates, $R^{\mathrm{L}}$, and of the axial and rhombic coefficients of the POMT, $c_{0}^{2}$ and $c_{2}^{2}$. Activation energies are derived from $R^{\mathrm{L}}$.

Methyl dynamics has been analyzed traditionally with the model-free (MF) approach. ${ }^{6}, 11^{-}$ 13 The formal MF analogue of $\tau=1 /\left(6 R^{\mathrm{L}}\right)$ is the effective local motional correlation time, $\tau_{\mathrm{e}}$. This parameter is typically inaccurate; in some cases, it is not even reported. ${ }^{20}$ Often $\tau_{\mathrm{e}}$ does not exhibit any temperature dependence. Therefore activation energies for local motion have only been reported for a small number of methyl groups studied over narrow temperature ranges. ${ }^{30}$ We show herein that $R^{\mathrm{L}}$ derived with SRLS exhibits Arrhenius-type temperature dependence over a substantial temperature range, enabling the determination of activation energies for virtually all the experimentally studied methyl groups of $\mathrm{Ca}^{2+}-\mathrm{CaM}^{*}$ smMLCKp. Information specific to the type of the methyl group is obtained. For example, we find that methionine methyl groups reorient on average six times faster, and have on average two times lower activation energies for local motion, than all the other 
methyl groups. The ultimate objective is to correlate the activation energies with the local structure.

Another physical quantity examined in detail in the present study is the POMT. Its form represents useful structural information. The first step, pursued herein, is to characterize the POMT and the associated local ordering tensor. The next step, to be pursued in future studies, is to correlate this information with the structure of the protein. Here, we show that the local motional rates and their activation energies, as well as the form and characteristics of the POMT, provide a clear and insightful mesoscopic picture of structural methyl dynamics in proteins.

The SRLS time correlation functions (TCFs) are obtained by solving numerically the twobody Smoluchowski equation. $17^{-19}$ The measurable spectral densities are obtained by linearly combining the generic spectral densities (which are Fourier Transforms of the TCFs) in accordance with the local geometry. The SRLS TCFs comprise a sum of multiple weighted exponents that correspond to the eigenmodes of motion. The weighting factors, which represent the contribution of each eigenmode, depend upon the local and global diffusion tensors, and the POMT. We illustrate herein typical SRLS TCFs for methyl dynamics and compare with the MF TCF. Note that TCFs for restricted motions in locally ordered media are also given by sums of multiple weighted exponents; 31 the respective theories, and SRLS, are based on the same principles (cf. ref. 19).

Finally, we examine the nature of the MF spectral density used to treat methyl dynamics, and the significance of the parameters that enter it. The main qualifier is $S_{\text {axis }}{ }^{2}$, interpreted as the amplitude of the $\mathrm{C}-\mathrm{CH}_{3}$ fluctuations. This parameter is given by the ratio of a squared "generalized"; order parameter, $S^{2}$, and a trigonometric coefficient, $0.1,25$ associated with a frame transformation.19 However, in the classical theories for treating restricted motions in liquids (e.g., refs $14^{-} 16$ and 31 ), order parameters (or more precisely tensors) are defined in terms of orienting potentials. They assess the preferential orientation of the local ordering frame in the (local) director frame; this represents important structural information. The quantities $S$ and $S_{\text {axis }}$ are artificial parameters both by definition ${ }^{22}$ and in practice, because they absorb unaccounted for effects. ${ }^{28,29}$ Therefore, their physical meaning is vague.

Three groups of $S_{\text {axis }}{ }^{2}$ values, centered at 0.2, 0.4 and 0.8 , have been observed in MF analyses. ${ }^{32}$ This classification has been interpreted as a manifestation of the microscopic nature of entropy in proteins. ${ }^{32}$ We show that these groups of $S_{\text {axis }}{ }^{2}$ values are just associated with different forms of the POMT, which the SRLS analysis effectively correlates with different methyl types.

We give a short Theoretical Background section followed by Results and Discussion, and then Conclusions.

\section{Theoretical Background}

\subsection{The slowly relaxing local structure (SRLS) approach}

The fundamentals of the stochastic coupled rotator slowly relaxing local structure theory, as applied to NMR spin relaxation in proteins, have been presented and reviewed previously. ${ }^{17-19}$ The application to methyl dynamics is described in refs 28 and ${ }^{29}$. A brief summary is given below for convenience.

The SRLS frames for methyl dynamics are shown in Figure 1a. L is the space-fixed laboratory frame with its Z-axis aligned along the external magnetic field, $B_{0}$. C is the global diffusion frame fixed in the protein. In this study, we consider the latter to be isotropic; hence, $\mathrm{C}$ also serves as local director. $\mathrm{Q}$ is the (axial) magnetic quadrupolar frame fixed in 
the probe. $\mathrm{M}$ is the (rhombic) local ordering/local diffusion frame fixed in the probe. The orientation of the $\mathrm{M}$ frame is not known a priori. In general, it is determined by data fitting. Figure $1 \mathrm{~b}$ illustrates the scenario wherein $\beta_{\mathrm{MD}}=110.5^{\circ}$ and $\gamma_{\mathrm{MD}}=0^{\circ}$. Within a good approximation, this represents "X-ordering", with the main ordering axis lying along the $\mathrm{C}$ $\mathrm{CDH}_{2}$ bond.

The time-dependent Euler angles, $\Omega_{\mathrm{LM}}(t)$, are modulated by the local and global motions. The time-dependent Euler angles, $\Omega_{\mathrm{LC}}(t)$, are modulated by the global tumbling. For describing the local motion we use a relative (probe versus protein) coordinate scheme; that is, $\Omega_{\mathrm{CM}}(t)=\Omega_{\mathrm{LM}}(t)-\Omega_{\mathrm{LC}}(t) .{ }^{18,19}$ The two rotators are coupled by the POMT, $U\left(\Omega_{\mathrm{CM}}\right)$. The diffusion equation for the coupled system is given by:

$$
\frac{\partial}{\partial t} P(X, t)=-\widehat{\Gamma} P(X, t)
$$

where $X$ is a set of coordinates completely describing the system. One has: ${ }^{18,19}$

$X=\left(\Omega_{\mathrm{CM}}, \Omega_{\mathrm{LC}}\right)$

$\widehat{\Gamma}=\widehat{\mathbf{J}}\left(\Omega_{\mathrm{CM}}\right) \mathbf{R}^{\mathrm{L}} P_{\mathrm{eq}} \widehat{\mathbf{J}}\left(\Omega_{\mathrm{CM}}\right) P_{\mathrm{eq}}^{-1}+\widehat{[\mathbf{J}}\left(\Omega_{\mathrm{CM}}\right)-\widehat{\mathbf{J}}\left(\Omega_{\mathrm{LC}}\right] \mathbf{R}^{\mathrm{C}} P_{\mathrm{eq}}\left[\widehat{\mathbf{J}}\left(\Omega_{\mathrm{CM}}\right)-\widehat{\mathbf{J}}\left(\Omega_{\mathrm{LC}}\right)\right] P_{\mathrm{eq}}^{-1}$,

where $\hat{\mathrm{J}}\left(\Omega_{\mathrm{CM}}\right)$ and $\hat{\mathrm{J}}\left(\Omega_{\mathrm{LC}}\right)$ are the infinitesimal rotation operators for the probe and the protein, respectively.

The Boltzmann distribution is $P_{\mathrm{eq}}=\exp \left[-U\left(\Omega_{\mathrm{CM}}\right) / k_{\mathrm{B}} T\right] /<\exp \left[-U\left(\Omega_{\mathrm{CM}}\right) / k_{\mathrm{B}} T\right]>$. The potential $U\left(\Omega_{\mathrm{CM}}\right)$ is in general expanded in the full basis set of the Wigner rotation matrix elements. In this study only the $L=2$ terms are preserved: ${ }^{18,19,28,29}$

$u\left(\Omega_{\mathrm{CM}}\right)=\frac{U\left(\Omega_{\mathrm{CM}}\right)}{k_{\mathrm{B}} T} \approx-c_{0}^{2} D_{0,0}^{2}\left(\Omega_{\mathrm{CM}}\right)-c_{2}^{2}\left[D_{0,2}^{2}\left(\Omega_{\mathrm{CM}}\right)+D_{0,-2}^{2}\left(\Omega_{\mathrm{CM}}\right)\right]$.

The coefficient $c_{0}^{2}$ is related to the strength of the POMT, and $c_{2}^{2}$ is related to its non-axiality. The order parameters, $S_{0}^{2}=\left\langle D_{00}^{2}\left(\Omega_{\mathrm{CM}}\right)>\right.$ and $S_{2}^{2}=<D_{02}^{2}\left(\Omega_{\mathrm{CM}}\right)+D_{0-2}^{2}\left(\Omega_{\mathrm{CM}}\right)>$ are calculated from $c_{0}^{2}$ and $c_{2}^{2}$ according to: ${ }^{18,19}$

$\left\langle D_{0 n}^{2}\left(\Omega_{\mathrm{CM}}\right)\right\rangle=\int d \Omega_{\mathrm{CM}} D_{0 n}^{2}\left(\Omega_{\mathrm{CM}}\right) \exp \left[-u\left(\Omega_{\mathrm{CM}}\right)\right] / \int d \Omega_{\mathrm{CM}} \exp \left[-u\left(\Omega_{\mathrm{CM}}\right)\right]$.

The Cartesian ordering tensor components are given by

$S_{z z}=S_{0}^{2}, S_{x x}=\left(\sqrt{3 / 2} S_{2}^{2}-S_{0}^{2}\right) / 2, S_{y y}=-\left(\sqrt{3 / 2} S_{2}^{2}+S_{0}^{2}\right) / 2$, with $S_{x x}+S_{y y}+S_{z z}=0$.

Expansion terms corresponding to $L=4, K=0,2,4,\left(c_{0}^{4}, c_{2}^{4}\right.$ and $\left.c_{4}^{4}\right)$ are included in our most recent computational scheme. ${ }^{33}$ They allow a more detailed modeling, in particular diffusion within two wells with less frequent jumps between them. ${ }^{15,34}$ More general jump models and modeling may be included by adding $U\left(\Omega_{\mathrm{CM}}\right)$. This is relevant for more complex methyl dynamics, to be considered in future work.

Equation 2 is solved to yield the SRLS time correlation functions which lead by Fourier transformation to the spectral densities, ${ }_{K, K^{\prime}}(\omega)=\sum_{i} \frac{c_{K, K^{\prime}, i} \tau_{i}}{1+\omega^{2} \tau_{i}^{2}} .{ }^{18,19}$ The relevant pairs, $K, K^{\prime}$, 
are determined by the symmetry of the local ordering/local diffusion and magnetic tensors. In practice, a finite number of terms is sufficient for numerical convergence of the solution.

The $j_{K, K^{\prime}}(\omega)$ functions are assembled into the measurable spectral density, $J^{\mathrm{QQ}}(\omega)$, according to the local geometry. ${ }^{17-} 19$ For ${ }^{2} \mathrm{H}$ relaxation in the presence of a rhombic POMT only six pairs, $K, K^{\prime}=0,0 ; 1,1 ; 2,2 ; 2,0 ; 1,-1$ and $2,-2$, persist. One has: ${ }^{28,29}$

$$
\begin{aligned}
J^{\mathrm{QQ}}(\omega) & =\left(d_{00}^{2}\left(\beta_{\mathrm{MQ}}\right)\right)^{2} j_{00}(\omega) \\
& +2\left(d_{10}^{2}\left(\beta_{\mathrm{MQ}}\right)\right)^{2} j_{11}(\omega) \\
& +2\left(d_{20}^{2}\left(\beta_{\mathrm{MQ}}\right)\right)^{2} j_{22}(\omega) \\
& +4 d_{00}^{2}\left(\beta_{\mathrm{MQ}}\right) d_{20}^{2}\left(\beta_{\mathrm{MQ}}\right) j_{02}(\omega) \\
& +2 d_{-10}^{2}\left(\beta_{\mathrm{MQ}}\right) d_{10}^{2}\left(\beta_{\mathrm{MQ}}\right) j_{-11}(\omega) \\
& +2 d_{-20}^{2}\left(\beta_{\mathrm{MQ}}\right) d_{20}^{2}\left(\beta_{\mathrm{MQ}}\right) j_{-22}(\omega),
\end{aligned}
$$

Together with the magnitude of the quadrupolar interaction, the spectral density values $J^{\mathrm{QQ}}(0), J^{\mathrm{QQ}}\left(\omega_{\mathrm{D}}\right)$ and $J^{\mathrm{QQ}}\left(2 \omega_{\mathrm{D}}\right)$ determine the experimentally measured relaxation rates ${ }^{2} \mathrm{H}$ $T_{1}$ and $T_{2}$, according to standard expressions for NMR spin relaxation. ${ }^{11}$ The fitting scheme for SRLS used in this study is described in ref 18 .

\subsection{Model-Free}

The MF approach provides directly the measurable spectral density, $J(\omega)$. The latter comprises two Lorentzian terms which represent the global motion and a single (effective) local motion. ${ }^{22,23}$ This simple form is based on the premise that the motions considered are independent statistically because they are time scale separated. $J(\omega)$ is given in MF by: ${ }^{22}$

$$
J(\omega)=S^{2} \tau_{\mathrm{m}} /\left(1+\tau_{\mathrm{m}}^{2} \omega^{2}\right)+\left(1-S^{2}\right) \tau_{\mathrm{e}} /\left(1+\tau_{\mathrm{e}}^{2} \omega^{2}\right),
$$

where $\tau_{\mathrm{m}}$ is the correlation time for the global motion, $\tau_{\mathrm{e}}<<\tau_{\mathrm{m}}$ is the effective correlation time for the local motion, and $S^{2}$ is the square of a "generalized" order parameter. By virtue of $\tau_{\mathrm{e}}<<\tau_{\mathrm{m}}$, one has $1 / \tau_{\mathrm{e}}{ }^{1}=1 / \tau_{\mathrm{m}}+1 / \tau_{\mathrm{e}} \sim 1 / \tau_{\mathrm{e}} . S^{2}$ is defined as the plateau value, $C^{\mathrm{L}}(\infty)$, to which the local motional time correlation function, $C^{\mathrm{L}}(t)$, is assumed to converge at long times. Mathematically $C^{\mathrm{L}}(\infty)$ is given by $\sum_{m=0, \pm 1, \pm 2}<\left|Y_{2 m}(\theta, \phi)\right|^{2}$, where $Y_{2 \mathrm{~m}}$ are the spherical harmonics of Brink and Satchler. $35 S \equiv \sqrt{C^{\mathrm{L}}(\infty)}$ is the "generalized" order parameter.

$S^{2}$ is considered to represent the amplitude of the local motion. In the context of the physical definition of order parameters (cf. eq 4), this is appropriate in the limit of a strong axial POMT, with the local motion in the extreme motional narrowing limit. ${ }^{16}$ Based on the theory of moments, $\tau_{\mathrm{e}}$ is defined as the area of the exact time correlation function for internal motion divided by $\left(1-S^{2}\right)$.

The extended MF (EMF) spectral density ${ }^{24}$ features a fast local motion, with effective correlation time, $\tau_{\mathrm{f}}$, and squared order parameters, $S_{\mathrm{f}}{ }^{2}$, and a slow local motion, with effective correlation time, $\tau_{\mathrm{s}}$, and squared order parameter, $S_{\mathrm{s}}{ }^{2}$. All three dynamic modes, represented by $\tau_{\mathrm{m}}, \tau_{\mathrm{f}}$ and $\tau_{\mathrm{s}}$, are assumed to be decoupled from one another. In practice, $\tau_{\mathrm{s}}$ and $\tau_{\mathrm{m}}$ are allowed to occur on the same time scale. ${ }^{36}$ 
In its application to methyl dynamics, eq 6 has been re-interpreted to represent two local motions. This was accomplished by factoring $\mathrm{S}$ into $S=\left[P_{2}\left(\cos 110.5^{\circ}\right)\right] \times S_{\text {axis }}=0.316 \times S_{\text {axis }}$. $11,22,37$ The factor 0.316 is considered to be the order parameter for methyl rotation around the $\mathrm{C}-\mathrm{CH}_{3}$ bond, referencing the article of Woessner. ${ }^{25} S_{\text {axis }}$ represents the order parameter for axial fluctuations of the $\mathrm{C}-\mathrm{CH}_{3}$ bond. The correlation time, $\tau_{\mathrm{e}}$, is assigned to both local motions. The spectral density given by eq 6 is recast as:

$J^{\mathrm{QQ}}(\omega)=0.1 \times S_{\text {axis }}{ }^{2} \tau_{\mathrm{m}} /\left(1+\omega^{2} \tau_{\mathrm{m}}{ }^{2}\right)+\left(1-0.1 \times S_{\text {axis }}{ }^{2}\right) \tau_{\mathrm{e}} /\left(1+\omega^{2} \tau_{\mathrm{e}}{ }^{2}\right)$,

where $1 / \tau_{\mathrm{e}}{ }^{\prime}=1 / \tau_{\mathrm{e}}+1 / \tau_{\mathrm{m}} \sim 1 / \tau_{\mathrm{e}}$.

In the limit where $S_{\text {axis }}{ }^{2}=1$ eq 7 should yield Woessner's model ${ }^{25}$; let us examine this limit. Woessner's model treats diffusive (or jump-type) motion about an axis tilted at a fixed angle, $\beta$, from an axial magnetic frame. The (tilted) diffusion axis tumbles isotropically with correlation time $\tau_{\mathrm{c}}$, the internal motion around it occurs with correlation tine, $\tau$, and $\tau_{\mathrm{c}} \gg \tau$. For diffusive internal motion one has two local motional decay constants, $\left(\tau{ }_{1}\right)^{-1}=\tau_{\mathrm{c}}{ }^{-1}+$ $\tau^{-1}$ and $\left(\tau_{2}^{\prime}\right)^{-1}=\tau_{\mathrm{c}}{ }^{-1}+4 \tau^{-1}$ for symmetrical jumps one has $\left(\tau^{\prime}\right)^{-1}=\tau_{\mathrm{c}}^{-1}+\tau^{-1}$. For $\beta=$ $110.5^{\circ}$, the measurable spectral density for diffusive motion is given by: $:^{25}$

$J^{\mathrm{QQ}}(\omega)=0.1 \tau_{\mathrm{c}} /\left(1+\omega^{2} \tau_{\mathrm{c}}{ }^{2}\right)+0.323 \tau_{1} /\left(1+\omega^{2}\left(\tau_{1}\right)^{2}\right)+0.577 \tau_{2} /\left(1+\omega^{2}\left(\tau_{2}\right)^{2}\right)$

$\left(d_{00}^{2}\left(110.5^{\circ}\right)\right)^{2}=0.1,2\left(d_{01}^{2}\left(110.5^{\circ}\right)\right)^{2}=0.323$ and $2\left(d_{02}^{2}\left(110.5^{\circ}\right)\right)^{2}=0.577$ are the elements of the (reduced) matrix representation of the Wigner rotation from the (axial) diffusion frame to the (axial) quadrupolar frame. The isotropic tumbling limit, $J^{\mathrm{QQ}}(\omega)=\tau_{\mathrm{c}} /\left(1+\omega^{2} \tau_{\mathrm{c}}{ }^{2}\right)$, is obtained for $\tau \rightarrow \infty, \beta_{\mathrm{MQ}} \rightarrow 0$, or both. ${ }^{25}$

In analogy with eq 8 , eq 7 has to fulfill the relation $\tau_{\mathrm{m}} \gg \tau_{\mathrm{e}}$; it should not be used when $\tau_{\mathrm{e}}$ and $\tau_{\mathrm{m}}$ are comparable in magnitude. This is implicit in MF, but is often not appreciated. ${ }^{36}$ Equation 7 does not converge to the isotropic tumbling limit of Woessner's model; rather, it yields $J^{\mathrm{QQ}}(\omega)=0.1 \tau_{\mathrm{c}} /\left(1+\omega^{2} \tau_{\mathrm{c}}{ }^{2}\right)$ for $S_{\text {axis }}{ }^{2}=1$ and $\tau_{\mathrm{e}} \rightarrow 0$. The parameter $\tau_{\mathrm{e}}$ represents simultaneously the internal motion in Woessner's model $(\tau)$, and restricted axial fluctuations of the $\mathrm{C}-\mathrm{CH}_{3}$ axis. The motion of this axis represents in Woessner's model the motion of the protein $\left(\tau_{\mathrm{c}}\right)$; so does $\tau_{\mathrm{m}}$ in eq 7 . Clearly, $\tau_{\mathrm{e}}$ is a physically vague parameter. The coefficient

0.1 in eq 7 is the trigonometric expression $\left(d_{00}^{2}(110.5)\right)^{2}$. It is not an order parameter; Woessner's model does not feature an order parameter. ${ }^{25}$ Clearly, $S_{\text {axis }}{ }^{2}=S^{2} / 0.1$, where $S$ is the "generalized" order parameter and 0.1 a geometric coefficient, is a physically vague quantity.

Equation 7 may not be considered to represent two restricted local motions. It represents a single diffusive local motion occurring in the presence of a weak axial POMT, with the main local ordering/local diffusion axis tilted at $110.5^{\circ}$ from the quadrupolar frame. For this scenario one has (e.g., cf. ref 19):

$$
\begin{gathered}
j_{00}(\omega)=\left(S_{0}^{2}\right)^{2} \tau_{\mathrm{m}} /\left(1+\omega^{2} \tau_{\mathrm{m}}{ }^{2}\right)+\left(1-\left(S_{0}^{2}\right)^{2} \tau_{0} /\left(1+\omega^{2} \tau_{0}{ }^{2}\right),\right. \\
j_{11}(\omega)=\tau_{1} /\left(1+\omega^{2} \tau_{1}{ }^{2}\right),
\end{gathered}
$$


and

$$
j_{22}(\omega)=\tau_{2} /\left(1+\omega^{2} \tau_{2}^{2}\right)
$$

The measurable spectral density is given by:

$J^{\mathrm{QQ}}(\omega)=\left(d_{00}^{2}\left(110.5^{\circ}\right)\right)^{2} j_{00}(\omega)+2\left(d_{01}^{2}\left(110.5^{\circ}\right)\right)^{2} j_{11}(\omega)+2\left(d_{02}^{2}\left(110.5^{\circ}\right)\right)^{2} j_{22}(\omega)=0.1 j_{00}(\omega)+0.323 j_{11}(\omega)+0.577 j_{22}(\omega)$

$(10)$

Assuming that $\tau_{0}=\tau_{1}=\tau_{2}=\tau$ (by virtue of $\tau_{\mathrm{m}} \gg \tau_{\mathrm{K}}$ ), one obtains:

$J^{\mathrm{QQ}}(\omega)=0.1 \times\left(S_{0}^{2}\right)^{2} \tau_{\mathrm{m}} /\left(1+\omega^{2} \tau_{\mathrm{m}}{ }^{2}\right)+\left(1-0.1 \times\left(S_{0}^{2}\right)^{2}\right) \tau /\left(1+\omega^{2} \tau^{2}\right)$.

This is formally the same as eq 7 wherein $S_{\text {axis }}{ }^{2}$ is replaced by $\left(S_{0}^{2}\right)^{2}$ and $\tau_{\mathrm{e}}$ is replaced by $\tau$. However, eq 11 is not suitable for describing methyl dynamics from a physical point-ofview, because a weak axial POMT implies a broad axial distribution of the instantaneous orientation of the $\mathrm{C}-\mathrm{CH}_{3}$ bond about the equilibrium orientation of the $\mathrm{C}-\mathrm{CH}_{3}$ bond. This is difficult to rationalize for tightly packed protein cores. On the other hand, a weak but rhombic POMT, inherent in the SRLS description, is associated with a non-axial distribution which can be accommodated, and is consistent with experimental observations ${ }^{26}$.

\section{Results and Discussion}

${ }^{2} \mathrm{H} T_{1}$ and $T_{2}$ data of the ${ }^{13} \mathrm{CDH}_{2}$ methyl groups of $\mathrm{Ca}^{2+}-\mathrm{CaM}^{*}$ smMLCKp, acquired at 14.1 and $17.6 \mathrm{~T}$, and 288, 295, 308 and $316 \mathrm{~K},{ }^{20}$ were analyzed with SRLS. For a rhombic POMT and an isotropic local diffusion tensor, $\boldsymbol{R}^{\mathbf{L}, 28,29}$ the variables in the data-fitting process are $c_{0}^{2}, c_{2}^{2}$ and $R^{\mathrm{C}}=\tau / \tau_{\mathrm{m}}$. Unique results are obtained by requiring that the local motional rates, $1 /(6 \tau)$, obey the Arrhenius relation. The global motional correlation time, $\tau_{\mathrm{m}}$, was taken from ref 20 . For simplicity, the angle $\gamma_{\mathrm{MD}}$ was set equal to zero. The angle $\beta_{\mathrm{MQ}}$ was allowed to deviate from $110.5^{\circ}$ by $1-2$ degrees; these deviations account to some extent for the complexity of the local structure wherein the methyl group reorients. The errors in the various best-fit parameters are estimated to be on the order of $10 \%$. The statistical measure used in our calculations is the percentile value for $\chi^{2}$ distribution. The reduced $\chi^{2}$, defined as $\chi^{2} / d f$, where $d f$ denotes the number of degrees of freedom, was in most cases below the critical 0.1 and 0.05 values. ${ }^{38}$

\subsection{Local motional diffusion rates, activation energies, and the POMT}

Figure 2 shows the local motional correlation time, $\tau$, and the axial $\left(c_{0}^{2}\right)$ and rhombic $\left(c_{2}^{2}\right)$ coefficients of the POMT, as a function of temperature for the various methyl types. For methionines, $\tau$ ranges between 7.8 and $19.5 \mathrm{ps}$; for the other methyl groups it ranges between 25.7 and 249.3 ps. On average, the methionine methyls reorient 10 times faster than the other methyl groups. In the latter category, the isoleucine $\delta$ methyls have the smallest $\tau$ values. The uniformity of $\tau$ among the various methyl types increases with increasing temperature.

Figure 3 a shows the temperature dependence of the local motional rates, $R^{\mathrm{L}}=1 /(6 \tau)$. Virtually all the methyl groups reorient with rates that increase with increasing temperature. 
Figure $3 \mathrm{~b}$ shows the activation energies, $E_{\mathrm{a}}$, derived from the temperature dependence of $R^{\mathrm{L}}$ using the Arrhenius equation. The $E_{\mathrm{a}}$ values lie in the range of $10-27 \mathrm{~kJ} / \mathrm{mol}$. With the exception of methyl M76, which resides in the flexible inter-domain linker of $\mathrm{Ca}^{2+}-\mathrm{CaM}^{*} \mathrm{smMLCKp},{ }^{39}$ the activation energies for the methionine methyls are approximately $10 \mathrm{~kJ} / \mathrm{mol}$.

The average value of $E_{\mathrm{a}}$ is comparable in magnitude to the average barrier height obtained for alanine methyls using molecular dynamics (MD) simulations. ${ }^{40}$ These barrier heights are difficult to determine accurately and unambiguously.40 The temperature dependence of $\tau_{\mathrm{e}}$ MF is in most cases not amenable to analysis in terms of activation energies. Reference ${ }^{20}$ does not report the $\tau_{\mathrm{e}}$ values; we calculated them using a version of Moldelfree 4.0 (ref 41) adapted to methyl dynamics. The results obtained for the methionine methyls (which are perhaps the best candidates for normal temperature dependence) are shown in Figure 4. The corresponding $\tau$ values obtained with SRLS are shown in Figure 5. Clearly, physically meaningful information cannot be derived from the data shown in Figure 4. On the other hand, data such as those shown in Figure 5 led to accurate activation energies for virtually all the methyl groups of $\mathrm{Ca}^{2+}-\mathrm{CaM}^{*} \mathrm{smMLCKp}$ (cf. Figure 3). Reference ${ }^{30}$ is among the few MF studies that reported activation energies for methyl group motion in proteins. This was accomplished for selected methyl groups examined over a temperature range of $17^{\circ} .30$ Although on average there was agreement with $\mathrm{MD},{ }^{40}$ site-specific agreement was not obtained.

The strength of the POMT is given by the coefficient $c_{0}^{2}$, shown in Figure 2b; its deviation from axial symmetry is given by the coefficient $c_{2}^{2}$, shown in Figure 2c. The $c_{0}^{2}$ values are predominantly positive, whereas all the $c_{2}^{2}$ values are negative. The alanine methyls exhibit the largest asymmetry, whereas the methionine methyls exhibit the smallest asymmetry.

Figure 6 shows the ordering tensor components $S_{0}^{2}$ and $S_{2}^{2}$ (eqs 3 and eqs 4). The axial component, $S_{0}^{2}$, is mostly positive, in some cases negative, and negative at all the temperatures studied for M76 and $\mathrm{L}_{2} 116$. The rhombic component, $S_{2}^{2}$, is invariably negative. The data shown in Figures $2 b, c$ reveal significant variations in the form of the POMT at the methyl sites of the complex $\mathrm{Ca}^{2+}-\mathrm{CaM}^{*}$ smMLCKp.

Average $c_{0}^{2}$ and $c_{2}^{2}$ values for all the methyl groups of $\mathrm{Ca}^{2+}-\mathrm{CaM}^{*}$ smMLCKp at the various temperatures studied are shown in Table 1. Between 288 and $308 \mathrm{~K}$ the parameter $\left\langle c_{0}^{2}\right\rangle$ (the strength of the average POMT) decreases gradually, whereas $\left|<c_{2}^{2}>\right| \sim 0.7$. The ratio $\left|\left\langle c_{2}^{2}\right\rangle\right|\left\langle c_{0}^{2}\right\rangle \mid$ is a measure of potential rhombicity; this parameters increases gradually between 288 and $308 \mathrm{~K}$. At $320 \mathrm{~K}$ the average POMT shows a sudden increase in $\left\langle c_{0}^{2}\right\rangle$ and decrease in both $\left\langle c_{2}^{2}\right\rangle$ and $\left|\left\langle c_{2}^{2}\right\rangle\right|\left\langle c_{0}^{2}\right\rangle \mid$. This might stem from the distinctively small temperature dependences of $c_{0}^{2}$ and $c_{2}^{2}$ for the methionine methyls (Figures $2 \mathrm{~b}, \mathrm{c}$ ).

Table 1 also shows the average $c_{0}^{2}$ and $c_{2}^{2}$ values for alanine, methionine and the other methyl types at $295 \mathrm{~K}$. The potential forms are different for these three methyl types, as shown conspicuously by the corresponding data in Table 1 . In Figure 7 we show the $\left|c_{2}^{2}\right|$ values obtained at $295 \mathrm{~K}$ as a function of methyl type. Values of $\left|c_{2}^{2}\right|$ of 1 and larger correspond exclusively to alanines, $\left|c_{2}^{2}\right|$ values of 0.5 and lower correspond exclusively to methionines, and in-between values correspond to all the other methyl types. 
The alanine methyls are attached directly to the protein backbone. The local structure at the site of their motion is likely to be affected significantly by the structural properties of the backbone; there is experimental evidence for this. ${ }^{42}$ It is thus reasonable that the POMT of the alanine methyls exhibits significant deviation from axiality, manifested as large $\left|c_{2}^{2}\right|$ values. Four out of six threonine methyls also have large $\left|c_{2}^{2}\right|$ values (Figure 7), for similar reasons. The methionine methyls, $\mathrm{S}-\mathrm{CH}_{3}$, reside at the end of long side chains; they are well separated from the backbone, and partly hydrophilic due to the sulphur atom. Methionine methyls move significantly faster than all the other methyl types (Figure 3a). These

observations are consistent with relative small deviation from axiality, i.e., small $\left|c_{2}^{2}\right|$ values

(Figure 7), and relatively small variations in $c_{2}^{2}$ throughout the temperature range investigated (Figure 2c). The isoleucine $\delta$ methyls, which are also attached to relatively long side chains, have likewise small $\left|c_{2}^{2}\right|$ values (Figure 7) and fast local motional rates (Figure $3 \mathrm{a})$. Thus, qualitative correlation between the magnitude of $R^{\mathrm{L}}$ and the asymmetry of the POMT on the one hand, and methyl type on the other hand, has been established effectively within the scope of the SRLS analysis.

It was reported that $S_{\text {axis }}{ }^{2}$ values of $\mathrm{Ca}^{2+}-\mathrm{CaM}^{*} \mathrm{smMLCKp} 32$ (and other proteins43) cluster into three groups centered at the values of $0.2,0.4$ and 0.8 . This has been interpreted as manifestation of the microscopic nature of entropy in proteins.32 We show in Figure 7 the MF $S_{\text {axis }}{ }^{2}$ values of the various methyl types of $\mathrm{Ca}^{2+}-\mathrm{CaM}^{*}$ smMLCKp, along with their $\left|c_{2}^{2}\right|$ values. It can be seen that $S_{\text {axis }}{ }^{2}$ values on the order of $0.8,0.2$ and 0.4 are associated with different extents of non-axiality of the POMT, which the SRLS analysis has correlated with alanines, methionines, and the other methyl types, respectively. The MF-based association is empirical in nature and does not connect with the methyl type. Correlation with entropic properties requires redefining $S_{\text {axis }}$ ad hoc from the original expression of $\sqrt{C^{\mathrm{L}}(\infty)} / 0.1$, to the physical expression of eq $4 .{ }^{32}$ In addition, one has to guess the form of $u\left(\Omega_{\mathrm{CM}}\right)$ in the Boltzmann factor that enters eq 4. The SRLS-based rationalization is straightforward within the scope of a general stochastic model for treating methyl dynamics.

\subsection{Typical POMTs and associated relative probability distributions}

We show in Figures 8a,c the local potentials, $u\left(\Omega_{\mathrm{CM}}\right)$ (cf. eq 3), obtained at $295 \mathrm{~K}$ for the methyl groups A10 and I885, plotted as a function of the angles $\beta_{\mathrm{CM}}$ and $\gamma_{\mathrm{CM}}$ in radians. Note that $u$ is the actual potential, $U$, divided by $k_{\mathrm{B}} T$, rendering $u$ dimensionless. The Figures $8 \mathrm{~b}, \mathrm{~d}$ show the corresponding relative (or unnormalized) probability distribution function, $P_{\text {rel }}=\exp (-u) \sin \beta_{\mathrm{CM}} \Delta \beta_{\mathrm{CM}} \Delta \gamma_{\mathrm{CM}}$. $P_{\text {rel }}$ is the probability of the main local ordering axis having an orientation in the infinitesimal ranges $\beta_{\mathrm{CM}} \pm \Delta \beta_{\mathrm{CM}}$ and $\gamma_{\mathrm{CM}} \pm \Delta \gamma_{\mathrm{CM}}$ for any $\alpha_{\mathrm{CM}}$ (since the $\mathrm{C}$ frame is uniaxial). ${ }^{44}$ Representations of the functions $P_{\text {rel }}\left(\beta_{\mathrm{CM}}, \gamma_{\mathrm{CM}}\right)$ are obtained by plotting $\mathrm{Z}_{\mathrm{C}}=R \cos \beta_{\mathrm{CM}}$ versus $\mathrm{X}_{\mathrm{C}}=R \sin \beta_{\mathrm{CM}} \cos \gamma_{\mathrm{CM}}$ and $\mathrm{Y}_{\mathrm{C}}=R \sin \beta_{\mathrm{CM}} \sin \gamma_{\mathrm{CM}}$, where $R=\exp (-u) \operatorname{sinp} \beta_{\mathrm{CM}}$.

The best-fit parameters, obtained with the data fitting strategy outlined above, are $c_{0}^{2}=0.087, c_{2}^{2}=-1.089, \beta_{\mathrm{MQ}}=112^{\circ}$ and $\tau / \tau_{\mathrm{m}}=0.0067$ for A10, and $c_{0}^{2}=0.633, c_{2}^{2}=-0.480$, $\beta_{\mathrm{MQ}}=113^{\circ}$ and $\tau / \tau_{\mathrm{m}}=0.0051$ for I $\delta 85$. The rhombic potential prevailing at the site of the motion of the methyl A10 is shown in Figure 8a. Its rhombicity, $\mid c_{2}^{2} / c_{0}^{2}$, is equal to 12.6; this represents very large asymmetry. The Cartesian ordering tensor components calculated in terms of this potential, shown in Table 2, indicate that "Y-ordering" prevails at this methyl site. The shape of the corresponding $P_{\text {rel }}$ function, shown in Figure 8b, bears out this symmetry. 
The rhombic potential prevailing at the site of the motion of the methyl I $\delta 85$ is shown in Figure $8 \mathrm{c}$. The $\left|c_{2}^{2} / c_{0}^{2}\right|$ ratio is 0.76 , indicating smaller asymmetry of the local potential as compared to methyl A10. The Cartesian tensor components given in Table 2 indicate that "nearly planar X"Z ordering" prevails at this methyl site. The shape of the corresponding $P_{\text {rel }}$ function, shown in Figure 8d, bears out this symmetry.

In Fig. 1b, we illustrate "X-ordering". The SRLS analysis presented in this study shows that this is just an example. The POMT at methyl sites is sensitive to the combined effect of methyl type and local structure; it has to be determined for each case by data fitting. It will be interesting and useful to correlate the SRLS POMT, $u\left(\Omega_{\mathrm{CM}}\right)$, and the associated ordering tensor, $\boldsymbol{S}$, with both experimentally determined (with X-ray crystallography or NMR), and MD-simulated, protein structures.

The MF analysis yielded $S_{\text {axis }}{ }^{2}=0.84$ for A10 and $S_{\text {axis }}{ }^{2}=0.303$ for I $\delta 85$ at $295 \mathrm{~K}^{20}$ Thus, according to MF the $\mathrm{C}-\mathrm{CH}_{3}$ bond of A10 is highly ordered; this is expected. On the other hand, the $\mathrm{C}-\mathrm{CH}_{3}$ bond of I $\delta 85$ is involved in large-amplitude axial fluctuations. This is incompatible with the local stereochemistry and the packing properties of protein cores. According to SRLS the A10 and I 885 methyl groups, and in general all the methyl groups in $\mathrm{Ca}^{2+}-\mathrm{CaM}^{*}$ smMLCKp, reorient in the presence of weak rhombic local potentials. Different methyl groups experience different potential forms determined by their type, and the structure of their immediate surroundings.

\subsection{Eigenmodes}

The SRLS time correlation functions comprise sums of weighted exponents. The decay constants are the eigenvalues of the SRLS time evolution operator $\Gamma$; we denote them egv. The corresponding eigenvectors are the eigenmodes; we denote their respective weighting factors egm. In the following, we characterize TCF functions in terms of the significantly contributing egv/egm terms.

In its physical connotation, where $S^{2}=\left(S_{0}^{2}\right)^{2}$ and $\tau_{\mathrm{e}}=\tau=1 /\left(6 R^{\mathrm{L}}\right)$, the MF spectral density of eq 6 can be described as follows. The local motional eigenvalue is egv ${ }^{\mathrm{L}}=6$ (in units of $R^{\mathrm{L}}$ ) and its weighting factor is egm ${ }^{\mathrm{L}}=\left(1-S^{2}\right)$; the global motional eigenvalue is egv ${ }^{\mathrm{G}}=\left(\tau_{\mathrm{e}} / \tau_{\mathrm{m}}\right)$ $\times 6$ and its weighting factor is egm ${ }^{G}=S^{2}$. The labels " $G$ " and " $L$ " indicate that the Wigner functions of the "bare" degrees of freedom have been preserved. The fact that emg ${ }^{\mathrm{G}}=S^{2}$ (and perforce egm $\mathrm{m}^{\mathrm{L}}=\left(1-S^{2}\right)$ ) is implied by considering $S^{2}$ to be the same as $\left(S_{0}^{2}\right)^{2}$.

The data set comprising ${ }^{2} \mathrm{H} T_{1}$ and $T_{2}$ of the methyl A10, acquired at 14.1 and 17.6 T, 295 $\mathrm{K}$, is used as an example. MF analysis based on eq 6 yields $S^{2}=0.084$ and $\tau_{\mathrm{e}}=77 \mathrm{ps}$ (calculated by us with Modelfree $4.0^{41}$ adapted to methyl dynamics) as best-fit parameters, with $\tau_{\mathrm{m}}=11.81 \mathrm{~ns}$ from ref 20 . The egv/egm contributions are egv $\mathrm{G} / \mathrm{egm}^{\mathrm{G}}=0.039(0.084)$ and $\mathrm{egv}^{\mathrm{L}} / \mathrm{egm}^{\mathrm{L}}=6(0.916)$. Figure $9 \mathrm{a}$ shows the eigenmode weighting factors on the ordinate and the eigenvalues on the abscissa.

In the corresponding SRLS calculation we allowed $c_{0}^{2}$ and $R^{\mathrm{C}}$ to vary, fixing $\beta_{\mathrm{MQ}}=0$ and $c_{2}^{2}=0$. The parameter $c_{0}^{2}$ yields $\left(S_{0}^{2}\right)^{2}$, which is formally analogous to $S^{2}$, and $R^{\mathrm{C}}$ is the formal analogue of $\tau_{\mathrm{e}} / \tau_{\mathrm{m}}$. The best-fit parameters are $c_{0}^{2}=0.87$ (which yields $\left(S_{0}^{2}\right)^{2}=0.036$ and $R^{\mathrm{C}}=$ 0.0015 . These values were used as input to the calculation of $C(t)=C_{00}(t)$ (only the $K, K^{\prime}=$ 0,0 component contributes to $C(t)$ when $\beta_{\mathrm{MQ}}=0$ ). The egv (egm) contributions are 0.0355 (0.0376), $5.96(0.350), 5.63(0.5)$ and $6.93(0.094)$. The total fractional contribution is 0.9906 (we ignore the large number of eigenvalues with very small weighting factors, which 
contribute the 0.0094 fraction). Figure $9 \mathrm{~b}$ shows the contributing eigenmodes and their corresponding eigenvalues. We also show these data, along with the egv/egm contributions to the $C_{11}(t)$ and $C_{22}(t)$ functions associated with this calculation, in Table 3, to enable comparison with subsequent scenarios where the $K, K^{\prime}=1,1$ and 2,2 components also contribute.

In the large time scale separation, which holds true for methyl dynamics, the SRLS pair 0.0355 (0.0376) represents within a good approximation the global motion. Hence one may compare it with $0.039(0.084)$ in MF. The MF parameter $S^{2}$ has the value of 0.084 whereas the SRLS parameter $\left(S_{0}^{2}\right)^{2}$ has the value of 0.0376 . The time scale separation is $\tau_{\mathrm{e}} / \tau_{\mathrm{m}}=$ 0.0065 in MF and $\tau / \tau_{\mathrm{m}}=0.0059$ in SRLS. Thus, even though the time scale separations are large, and the axial local ordering and magnetic frames are collinear in both calculations, the squared order parameter (the local motional correlation time) is 2.2 (1.1) times larger in MF in comparison with SRLS. The large discrepancy between the order parameters is implied by the fact that the Wigner functions are no longer eigenfunctions of the local motional diffusion operator in the presence of a POMT given by $c_{0}^{2}=0.87$. This is ignored in MF, where a single decay constant for the local motion, $1 / \tau_{\mathrm{e}}$, representing a Wigner function scenario, is assumed allegedly. The more physically meaningful SRLS calculation shows that three main local motional eigenmodes with eigenvalues 5.96, 5.63 and 6.93, and corresponding weighting factors of $0.350,0.5$ and 0.094 , are present.

We proceed by accounting for the local geometry. This is done in MF implicitly through the coefficient 0.1 featured by eq 7 . This spectral density, which is a recasting of eq 6 , comprises egv ${ }^{\mathrm{G}} / \mathrm{egm}^{\mathrm{G}}=0.039(0.84)$ and $\mathrm{egv}^{\mathrm{L}} / \mathrm{egm}^{\mathrm{L}}=6(0.16)$. In the corresponding SRLS calculation we allowed $c_{0}^{2}$ and $R^{\mathrm{C}}$ to vary, fixing $\beta_{\mathrm{MQ}}=110.5^{\circ}$ and $c_{2}^{2}=0$. We obtained $c_{0}^{2}=2.97$ (which yields $\left.\left(S^{2}{ }_{0}\right)^{2}=0.36\right)$ and $R^{\mathrm{C}}=0.0009$ as best-fit values. The latter were used as input to the calculation of $C_{00}(t), C_{11}(t)$ and $C_{22}(t)$ (which are the relevant TCFs when the (axial) $\mathrm{M}$ and $\mathrm{Q}$ frames are tilted). The coefficients of these functions in the measurable TCF are $0.1,0.323$ and 0.577 (cf. eq 10); hence, the weighting factors are scaled when they enter the measurable time correlation function (or spectral density).

Table 4 features the unscaled weighting factors; the scaled weighting factors are shown in Figure $9 \mathrm{c}$ by circles, triangles and diamonds for the $C_{00}(t), C_{11}(t)$ and $C_{22}(t)$ contributions, respectively. Figures $9 \mathrm{c}$ and $9 \mathrm{~b}$ differ significantly. Clearly, the $\beta_{\mathrm{MQ}}=110.5^{\circ}$ geometry, hence Figure 9c, is correct. However, the results illustrated in Figure $9 \mathrm{c}$ are not acceptable because $c_{0}^{2}=2.97$ represents a weak axial POMT, which, as pointed out above, is not considered to be appropriate from a physical point-of-view.

SRLS analyses of methyl dynamics based on axial potentials yielded results with several problematic aspects. ${ }^{28,29}$ Many of these matters could be resolved by allowing for rhombic potentials. ${ }^{28,29}$ We therefore proceed by subjecting the experimental data of A10 to SRLS analysis where rhombic potentials are allowed for. For meaningful comparion with the axial potentials considered so far, we use results that feature "Z-ordering" and have the angle $\beta_{\mathrm{MQ}}$ closest to $110.5^{\circ}$. The corresponding best-fit values are $c_{0}^{2}=1.76, c_{2}^{2}=-0.59, R^{\mathrm{C}}=0.0054$ and $\beta_{\mathrm{MQ}}=109^{\circ}$. These values have been used as input to the calculation of the $C_{K K^{\prime}}(t)$ functions for $K, K^{\prime}=0,0 ; 1,1 ; 2,2 ; 2,0 ; 1,-1$ and $2,-2$ (cf. eq 5). The corresponding scaling factors are $0.14,0.354,0.474,-0.095,0.045$ and 0.082 . The scaled eigenmodes contributed by $C_{00}(t), C_{11}(t)$ and $C_{22}(t)$ are depicted in Figure $9 \mathrm{~d}$; the unscaled values are shown in Table 5 . 
Figure 9d differs significantly from Figure 9c. The calculation illustrated in Figure 9d, featuring a rhombic potential, is appropriate both from a physical point-of-view and from a statistical point-of-view (low $\chi^{2}$ ); hence, it is accepted. There is ample evidence for nonaxial restrictions underlying methyl dynamics in proteins. ${ }^{26-28,} 45-47$

Further insight into the effect of mode-coupling, general features of local geometry, and tensor symmetry, can be gained by careful comparison of corresponding data shown in Tables 3-5. As expected, mode-coupling has a small effect in methyl dynamics because the local motion of the methyl group is much faster than the global tumbling of the protein. On the other hand, as a result of a POMT which is rhombic, the solution of the Smoluchowski equation is no longer analytical, but rather yields multi-exponantial decaying time correlation functions.

The version of eq 6 in which $S^{2}=\left(S_{0}^{2}\right)^{2}$ and $\tau_{\mathrm{e}}=\tau=1 /\left(6 R^{\mathrm{L}}\right)$ constitutes the simplest physically sound description of the two-body problem. ${ }^{48}$ Equation 7 is a reformulation of eq 6. It cannot possibly represent a three-body problem with the two local motions necessarily related geometrically, but with no provision to account for geometric features, unless one resorts to the utilization of constructs ( $\tau_{\mathrm{e}}$ and $S_{\text {axis }}{ }^{2}$ ). The results are impaired thereby; ${ }^{27,28}$ even trends may be misleading. For example, $\left(S_{0}^{2}\right)^{2}=0.36$ whereas $S_{\text {axis }}^{2}=0.84$ for methyl A10, and $\left(S_{0}^{2}\right)^{2}=0.14$ whereas $S_{\text {axis }}{ }^{2}=0.11$ for methyl M76. The parameterizing values, $S_{\text {axis }}{ }^{2}$, span a significantly larger range than the physical values, $\left(S_{0}^{2}\right)^{2} ; 18$ the relation between $\left(S_{0}^{2}\right)^{2}$ and $S_{\text {axis }}{ }^{2}$ is not linear. ${ }^{29}$ Therefore, care is to be exerted in using $S_{\text {axis }}{ }^{2}$ and $\tau_{\mathrm{e}}$ to derive information considered biologically relevant. ${ }^{49-53}$

\subsection{Aspects of structural and functional dynamics}

Structural features-SRLS provides both the axial, $S_{0}^{2}$, and rhombic, $S_{2}^{2}$, order parameters (Figure 6; see above). The absolute values of $S_{0}^{2}$ and $S_{2}^{2}$ are small and comparable in magnitude. This signifies weak and highly rhombic local ordering. Different relative signs of $S_{0}^{2}$ and $S_{2}^{2}$ correspond to different rhombic symmetries. The same kind of information can be derived from the coefficients of the local potential, $c_{0}^{2}$ and $c_{2}^{2}$ (Figure $2 \mathrm{~b}, \mathrm{c})$. The orientation of the local ordering frame relative to the magnetic quadrupolar frame is given by the angle $\beta_{\mathrm{MQ}}$, which is within 1-2 degrees from $110.5^{\circ}$. This means that the main local ordering axis is nearly parallel to the $\mathrm{C}-\mathrm{CH}_{3}$ bond.

The structural information outlined above is mesoscopic in nature. Establishing correlations at the atom level between the SRLS parameters $S_{0}^{2}, S_{2}^{2}$ and $E_{\mathrm{a}}$, and structural features of the $\mathrm{Ca}^{2+}-$ calmodulin*smMLCKp complex has to await the development of appropriate integrated methodologies which combine mesoscopic and atomistic approaches. Such developments exist in different contexts; ${ }^{54-63}$ adaptation to NMR spin relaxation in proteins is in progress.

Molecular dynamics-The $\mathrm{Ca}^{2+}-$ calmodulin*smMLCKp complex was studied with MD simulations. ${ }^{64}$ Squared generalized order parameters, $S^{2}(\mathrm{MD})$, were calculated with a simple formula applicable to ultrafast local motions. ${ }^{65}$ As typically found,37,66,67 the parameter $S^{2}(\mathrm{MD})$ overestimates $S^{2}(\mathrm{MF})$. This is likely associated with different parameterization of the actual time correlation function in the MF and MD analyses. ${ }^{19}$ The only correlation established between $S^{2}(\mathrm{MD})$ and the 3D structure of $\mathrm{Ca}^{2+}$-calmodulin*smMLCKp is the proximity of the side chains of aromatic residues to a given methyl group. ${ }^{64}$ 
The alanine methyl groups of staphylococcal nuclease were investigated. ${ }^{40}$ It was found that infrequently sampled low-barrier conformations might affect the results significantly. Integrated approaches might be useful in this context.

Xue et al. studied methyl dynamics in the SH3 domain of $\alpha$-spectrin with both ${ }^{2} \mathrm{H}$ NMR relaxation analyzed with MF, and with $\mathrm{MD}{ }^{30}$ The MF analysis yielded an average barrier height of $2.8 \mathrm{kcal} / \mathrm{mol}$. The MD simulations yielded average barrier heights of $3.1-3.5 \mathrm{kcal} /$ mol. The analysis of several NMR structures from the Protein Data Base yielded average barrier heights of 4-6 kcal/mol. Integrated approaches might provide new insights.

Biological function-The effect of $\mathrm{Ca}^{2+}$ binding on the side-chain methyl dynamics of calbindin $\mathrm{D}_{9 \mathrm{k}}$ was studied. ${ }^{49}$ The results revealed correlation between a methyl group's distance from the $\mathrm{Ca}^{2+}$ binding site and its conformational dynamics. Two classes, one proximal and the other one distal, were identified according to the values of the best-fit MF parameters. It was inferred that the distal and proximal methyl groups respond differently to $\mathrm{Ca}^{2+}$ binding. A new trend, exhibited by the distal methyl groups, was linked to an important mechanism by which calbindin $\mathrm{D}_{9 \mathrm{k}}$ achieves high affinity binding while minimizing the corresponding loss of conformational entropy. The term "polar dynamics" was introduced.

Information on ligand-dependent dynamics and intramolecular signaling was obtained by analyzing with $\mathrm{MF}{ }^{2} \mathrm{H}$ methyl relaxation in a PDZ domain. ${ }^{50}$ This line of reasoning was pursued to suggest that position 20 in the human tyrosine phosphatase $1 \mathrm{E}$ protein acts as a critical "hub" for transmitting changes in dynamics. ${ }^{51}$ Side-chain methyl MF order parameters have been used to infer on hidden dynamic allostery in a PDZ domain. ${ }^{52}$

Since the the absolute values of the MF parameters and the variations therein are inaccurate, the biologically-relevent assessments mentioned above might change upon SRLS analysis of the same experimental data.

Conformational entropy-The parameter $S_{\text {axis }}$ has been used to derive conformational entropy in the context of ligand binding or temperature variations. ${ }^{6,32}$ As pointed out above, the accuracy of $S_{\text {axis }}$, and its physical meaning, are problematic. An axial potential, with an $a d$-hoc defined shape, is calculated from $S_{\text {axis }}$ and used further to calculate conformational entropy. In SRLS conformational entropy is calculated directly from the rhombic potential determined by physical data fitting. Since this potential is significantly more realistic than the axial MF potentials mentioned above, the SRLS-based entropy is more accurate and informative than the MF-based entropy. Information associated with the $\mathrm{Ca}^{2+}$-calmodulin*smMLCKp complex appears in ref 19 . We found that the variations in the experimental data are associated primarily with variations in the rhombicity of the local spatial restrictions represented by either $S_{2}^{2}$ or $c_{2}^{219}$ The overall picture provided by SRLS differs significantly from the overall picture provided by MF.

\section{Conclusions}

Local motional rates and associated activation energies are reported for all the experimentally studied methyl groups of $\mathrm{Ca}^{2+}-\mathrm{CaM}^{*}$ smMLCKp. In the temperature range of 288 to $320 \mathrm{~K}$ the local motional rates lie in the range $8.6 \times 10^{9}-21.4 \times 10^{9} 1 / \mathrm{s}$ for methionines, and $0.67 \times 10^{9}-6.5 \times 10^{9} 1 / \mathrm{s}$ for all the other methyl types. The activation energies for local motion are approximately $10 \mathrm{~kJ} / \mathrm{mol}$ for methionines, and $10-27 \mathrm{~kJ} / \mathrm{mol}$ for all the other methyl types. The potential of mean torque is weak and non-axial; its form differs for alanines, methionines and all the other methyl types. The measurable time correlation function represents predominantly the local motion. It comprises a relatively 
large number of eigenmodes resulting from the POMT, and its rhombic symmetry. The spectral density used in model-free analyses of methyl dynamics in proteins is physically vague; so are the parameters that enter it. The three classes of $S_{\text {axis }}{ }^{2}$ associated in the literature with the microscopic origin of the entropy in proteins are shown to be associated with different forms of the POMT. Both the activation energies and the POMT forms represent important information because they relate to the local structure. Well-defined physical rates for local methyl motion provide important kinetic information.

\section{References}

1. Ishima R, Torchia DA. Nat. Struct. Biol. 2000; 7:740. [PubMed: 10966641]

2. Case DA. Acc. Chem. Res. 2002; 35:325. [PubMed: 12069616]

3. Brüschweiler R. Curr. Opin. Struct. Biol. 2003; 13:175. [PubMed: 12727510]

4. Palmer AG. Chem. Rev. 2004; 104:3623. [PubMed: 15303831]

5. Mittermaier A, Kay LE. Science. 2006; 312:224. [PubMed: 16614210]

6. Igumenova TI, Frederick KK, Wand AJ. Chem. Rev. 2006; 106:1672. [PubMed: 16683749]

7. Kitao A, Wagner G. Mag. Reson. Chem. 2006; 44:S130.

8. Jarymowycz VA, Stone MJ. Chem. Rev. 2006; 106:1624. [PubMed: 16683748]

9. Nodet G, Abergel D. Eur. Biophys. J. Biophys. Lett. 2007; 36:985.

10. Markwick PRL, Malliavin T, Nilges M. PLoS Comput. Biol. 2008; 4:1.

11. Muhandiram DR, Yamazaki T, Sykes BD, Kay LE. J. Am. Chem. Soc. 1995; 117:11536.

12. Millet O, Muhandiram DR, Skrynnikov NR, Kay LE. J. Am. Chem. Soc. 2002; 124:6439.

[PubMed: 12033875]

13. Srynnikov NR, Millet O, Kay LE. J. Am. Chem. Soc. 2002; 124:6449. [PubMed: 12033876]

14. Polimeno A, Freed JH. Adv. Chem. Phys. 1993; 83:89.

15. Polimeno A, Freed JH. J. Phys. Chem. 1995; 99:10995.

16. Liang Z, Freed JH. J. Phys. Chem. B. 1999; 103:6384.

17. Tugarinov V, Liang Z, Shapiro Yu E, Freed JH, Meirovitch E. J. Am. Chem. Soc. 2001; 123:3055. [PubMed: 11457016]

18. Meirovitch E, Shapiro Yu E, Polimeno A, Freed JH. J. Phys. Chem. A. 2006; 110:8366. [PubMed: 16821820]

19. Meirovitch E, Shapiro Yu E, Polimeno A, Freed JH. Prog. NMR Spectrosc. 2010; 56:360.

20. Lee A, Sharp KA, Kranz JK, Song X-J, Wand AJ. Biochemistry. 2002; 41:13814. [PubMed: 12427045]

21. Kay LE, Torchia DA, Bax A. Biochemistry. 1989; 28:8972. [PubMed: 2690953]

22. Lipari G, Szabo A. J. Am. Chem. Soc. 1982; 104:4546.

23. Lipari G, Szabo A. J. Am. Chem. Soc. 1982; 104:4559.

24. Clore GM, Szabo A, Bax A, Kay LE, Driscoll PC, Gronenborn AM. J. Am. Chem. Soc. 1990; 112:4989.

25. Woessner DE. J. Chem. Phys. 1962; 36:1.

26. Avitabile J, London RE. J. Am. Chem. Soc. 1978; 100:7159.

27. Tugarinov V, Kay LE. J. Biomol. NMR. 2004; 29:369. [PubMed: 15213435]

28. Meirovitch E, Polimeno A, Freed JH. J. Phys. Chem. B. 2006; 110:20615. [PubMed: 17034251]

29. Meirovitch E, Shapiro Yu E, Polimeno A, Freed JH. J. Phys. Chem. B. 2007; 111:12865. [PubMed: 17941658]

30. Xue Y, Pavlova MS, Ryabov YE, Reif B, Skrynnikov NR. J. Am. Chem. Soc. 2007; 129:6827. [PubMed: 17488010]

31. Emsley JW, Luckhurst GR, Stockley CP. Proc. R. Soc. London Ser. A. 1982; 381:117.

32. Lee AL, Wand AJ. Nature. 2001; 411:501. [PubMed: 11373686]

33. Zerbetto M, Polimeno A, Meirovitch E. J. Phys. Chem. B. 2009; 113:13613. [PubMed: 19775101]

34. Moro GJ, Polimeno A. J. Chem. Phys. 1997; 107:7884.

J Phys Chem B. Author manuscript; available in PMC 2012 January 20. 
35. Brink, DM.; Satchler, GR. Angular Momentum. Oxford: Clarendon Press; 1968.

36. Xu J, Xue Y, Srynnikov NR. J. Biomol. NMR. 2009; 45:57. [PubMed: 19582374]

37. Chatfield DC, Szabo A, Brooks BR. J. Am. Chem. Soc. 1998; 120:5301.

38. Spiegel, MR.; Liu, J. Schaum's Outline Series. 2nd Ed.. New York: McGraw-Hill; 1999. Mathematical Handbook of Formulas and Tables; p. 266

39. Babu YS, Bugg CE, Cook WJ. J. Mol. Biol. 1988; 204:191. [PubMed: 3145979]

40. Chatfield DC, Augusten A, D’Cunha C. J. Biomol. NMR. 2004; 29:377. [PubMed: 15213436]

41. Mandel AM, Akke M, Palmer AG III. J. Mol. Biol. 1995; 246:144. [PubMed: 7531772]

42. Batchelder LS, Niu CH, Torchia DA. J. Am. Chem. Soc. 1983; 105:2228.

43. Lee AL, Kinnear SA, Wand A. J. Nat. Struct. Biol. 2000; 7:72.

44. Barnes JP, Freed JH. Biophys. J. 1998; 75:2532. [PubMed: 9788949]

45. Best RB, Clarke J, Karplus M. J. Mol. Biol. 2005; 349:185. [PubMed: 15876377]

46. Vugmeyster L, Ostrovsky D, Ford JJ, Burton SD, Lipton AS, Hoatson GL, Vold RL. J. Am. Chem. Soc. 2009; 131:13651. [PubMed: 19772361]

47. Tamura A, Matsushita M, Naito A, Kojima S, Miura KI, Akasaka K. Protein Science. 1996; 5:127. [PubMed: 8771205]

48. Freed JH. J. Chem. Phys. 1977; 66:4183.

49. Johnson E, Palmer AG III, Rance M. Proteins. 2007; 66:796. [PubMed: 17173286]

50. Fuentes EJ, Gilmore SA, Mauldin RV, Lee AL. J. Mol. Biol. 2006; 364:337. [PubMed: 17011581]

51. Clarkson MW, Gilmore SA, Edgell MH, Lee AL. Biochemistry. 2006; 45:7693. [PubMed: 16784220]

52. Law AB, Fuentes EJ, Lee AL. J. Am. Chem. Soc. 2009; 131:6322. [PubMed: 19374353]

53. Petit CM, Zhang J, Sapienza PJ, Fuentes EJ, Lee AL. Proc. Natl. Acad. Sci. 2009; 106:18249. [PubMed: 19828436]

54. Zerbetto M, Polimeno A, Kotsyubynskyy D, Ghalebani L, Kowalewski J, Meirovitch E, Olsson U, Widmalm G. J. Chem. Phys. 2009; 131:234501. [PubMed: 20025329]

55. Barone V, Polimeno A. PhysChemChemPhys. 2006; 8:4609.

56. Barone V, Brustolon M, Cimino P, Polimeno A, Zerbetto M, Zoleo A. J. Am. Chem. Soc. 2006; 128:15865. [PubMed: 17147399]

57. Zerbetto M, Carlotto S, Polimeno A, Corvaja C, Franco L, Toniolo C, Formaggio F, Barone V, Cimino P. J. Phys. Chem. B. 2007; 111:2668. [PubMed: 17311450]

58. Carlotto S, Cimino P, Zerbetto M, Franco L, Corvaja C, Crisma M, Formaggio F, Toniolo C, Polimeno A, Barone V. J. Am. Chem. Soc. 2007; 129:11248. [PubMed: 17705490]

59. Barone V, Polimeno A. Chem. Soc. Rev. 2007; 36:1724. [PubMed: 18213981]

60. Sezer D, Freed JH, Roux B. J. Am. Chem. Soc. 2009; 131:2597. [PubMed: 19191603]

61. Sezer D, Freed JH, Roux B. J. Chem. Phys. 2008; 128:165106. [PubMed: 18447510]

62. Sezer D, Freed JH, Roux B. J. Phys. Chem. B. 2008; 112:11014. [PubMed: 18698714]

63. Sezer D, Freed JH, Roux B. J. Phys. Chem. B. 2008; 112:5755. [PubMed: 18412413]

64. Prabhu NV, Lee AL, Wand AJ, Sharp KA. Biochemistry. 2003; 42:562. [PubMed: 12525185]

65. Henry E, Szabo A. J. Chem. Phys. 1985; 82:4753.

66. Hu H, Hermans J, Lee A. L. J. Biomol. NMR. 2005; 32:151. [PubMed: 16034666]

67. Showalter SA, Johnson E, Rance M, Brüschweiler R. J. Phys. Chem. B. 2008; 112:6203. [PubMed: 18376887] 


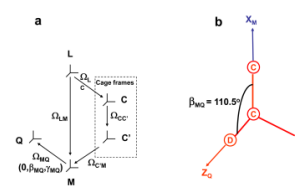

Figure 1.

(a) Various reference frames that define the SRLS model. L is the laboratory frame; $\mathrm{C}$ is the global diffusion frame, taken isotropic in this study; $\mathrm{M}$ is the local ordering/local diffusion frame fixed in the C-D bond; $\mathrm{Q}$ is the magnetic quadrupolar dipolar frame fixed in the $\mathrm{C}-\mathrm{D}$ bond; $\Omega_{\mathrm{LC}}$ are $\Omega_{\mathrm{CM}}$ are time-dependent Euler angles associated with the global motion and the relative local motion, respectively. (b) Methyl group schematic corresponding to a rhombic local ordering scenario with $\beta_{\mathrm{MQ}}=110.5^{\circ}$ and $\gamma_{\mathrm{MD}}=0^{\circ}$. Within a good approximation, this represents "X-ordering". The main ordering axis lies along the $\mathrm{C}-\mathrm{CDH}_{2}$ bond. 

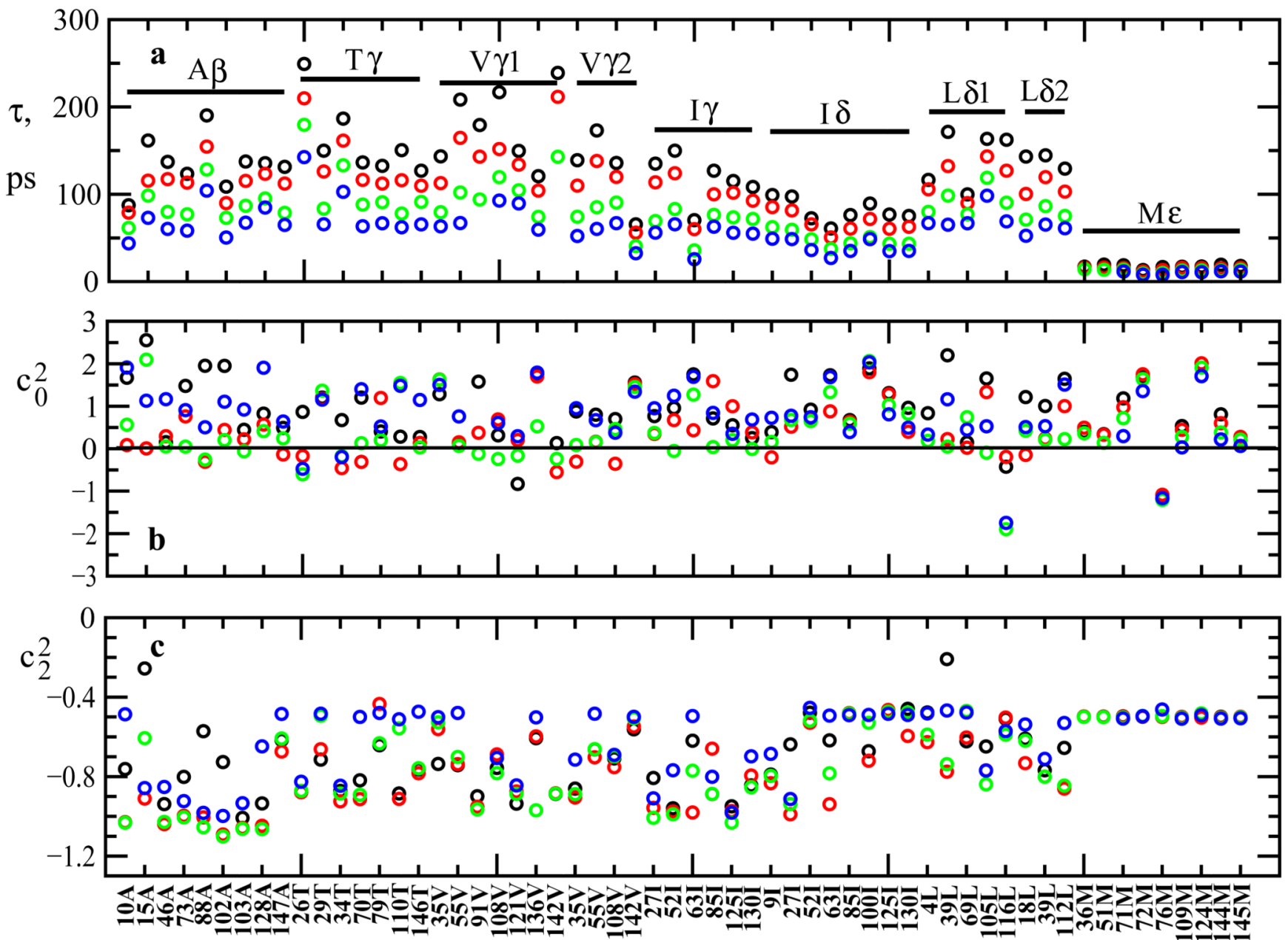

Figure 2.

Best-fit local motional correlation time, $\tau$ (a), axial potential coefficient, $c_{0}^{2}(\mathrm{~b})$, and rhombic potential coefficient, $c_{2}^{2}$ (c), as a function of methyl type at the various temperatures studied (black - 288, red -299 , green -308 , blue $-320 \mathrm{~K}$ ). Details on the fitting procedure are given in the text. 


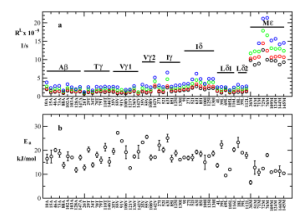

Figure 3.

(a) Best-fit local motional rate, $R^{\mathrm{L}}=1 /(6 \tau)$, as a function of methyl type, at the various temperatures studied (black - 288, red - 299, green - 308, blue - $320 \mathrm{~K}$ ). (b) Activation energies, $E_{\mathrm{a}}$, as a function of methyl type, calculated with the Arrhenius equation from the $R^{\mathrm{L}}$ values shown in part (a). 


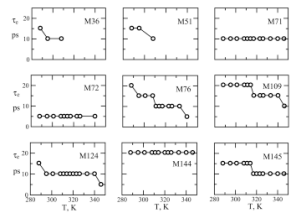

Figure 4.

Effective local motional correlation time, $\tau_{\mathrm{e}} \mathrm{MF}$, of the various methionine methyls of $\mathrm{Ca}^{2+}-\mathrm{CaM}^{*}$ smMLCKp, as a function of temperature. The relevant experimental data from ref 20 were analyzed with the program Modelfree $4.0^{41}$ adapted to methyl dynamics. Equation 7 was used; $S_{\text {axis }}^{2}$ and $\tau_{\mathrm{e}}$ were allowed to vary. 


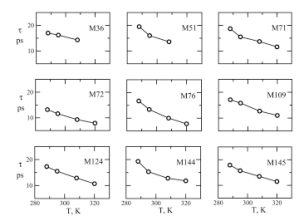

Figure 5.

Local motional correlation time, $\tau=1 /\left(6 R^{\mathrm{L}}\right)$ SRLS, of the various methionine methyls of $\mathrm{Ca}^{2+}-\mathrm{CaM}^{*}$ smMLCKp, as a function of temperature. The variables in these calculations were $c_{0}^{2}, c_{2}^{2}$ and $R^{\mathrm{L}} ; \beta_{\mathrm{MQ}}$ was allowed to depart from $110.5^{\circ}$ by $1-2$ degrees. 

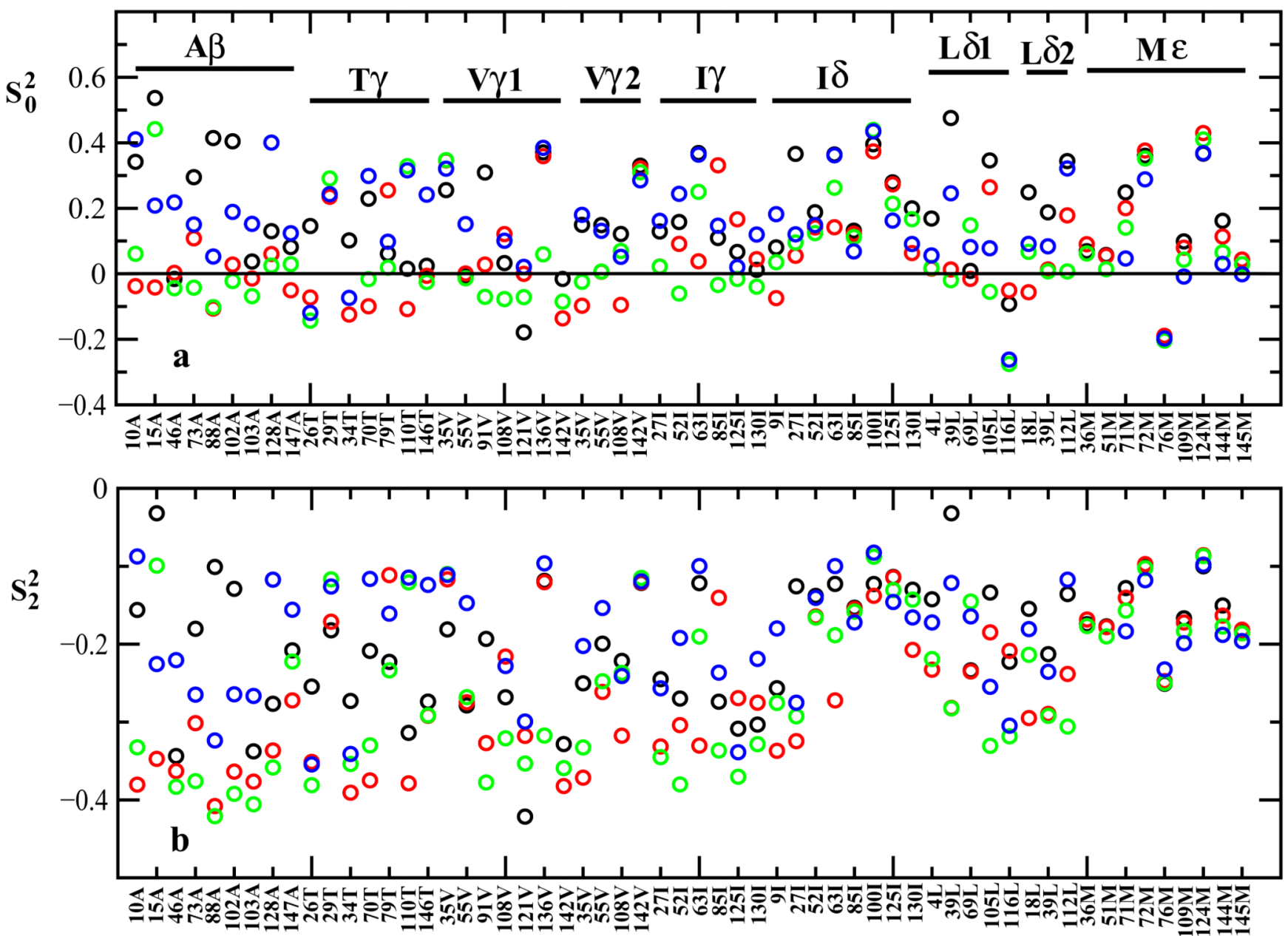

Figure 6.

Axial $S_{0}^{2}$ (a) and rhombic $S_{2}^{2}$ (b) ordering tensor components, obtained from the best-fit potential coefficients $c_{0}^{2}$ and $c_{2}^{2}$ (cf. Figures $2 \mathrm{~b}, \mathrm{c}$ ) using eq 3 and 4 , as a function of methyl type, at the various temperatures studied (black - 288, red - 299, green - 308, blue - $320 \mathrm{~K}$ ).

The variables in these calculations were $c_{0}^{2}, c_{2}^{2}$ and $R^{\mathrm{L}} ; \beta_{\mathrm{M} \mathrm{Q}}$ was allowed to depart from $110.5^{\circ}$ by $1-2$ degrees. 


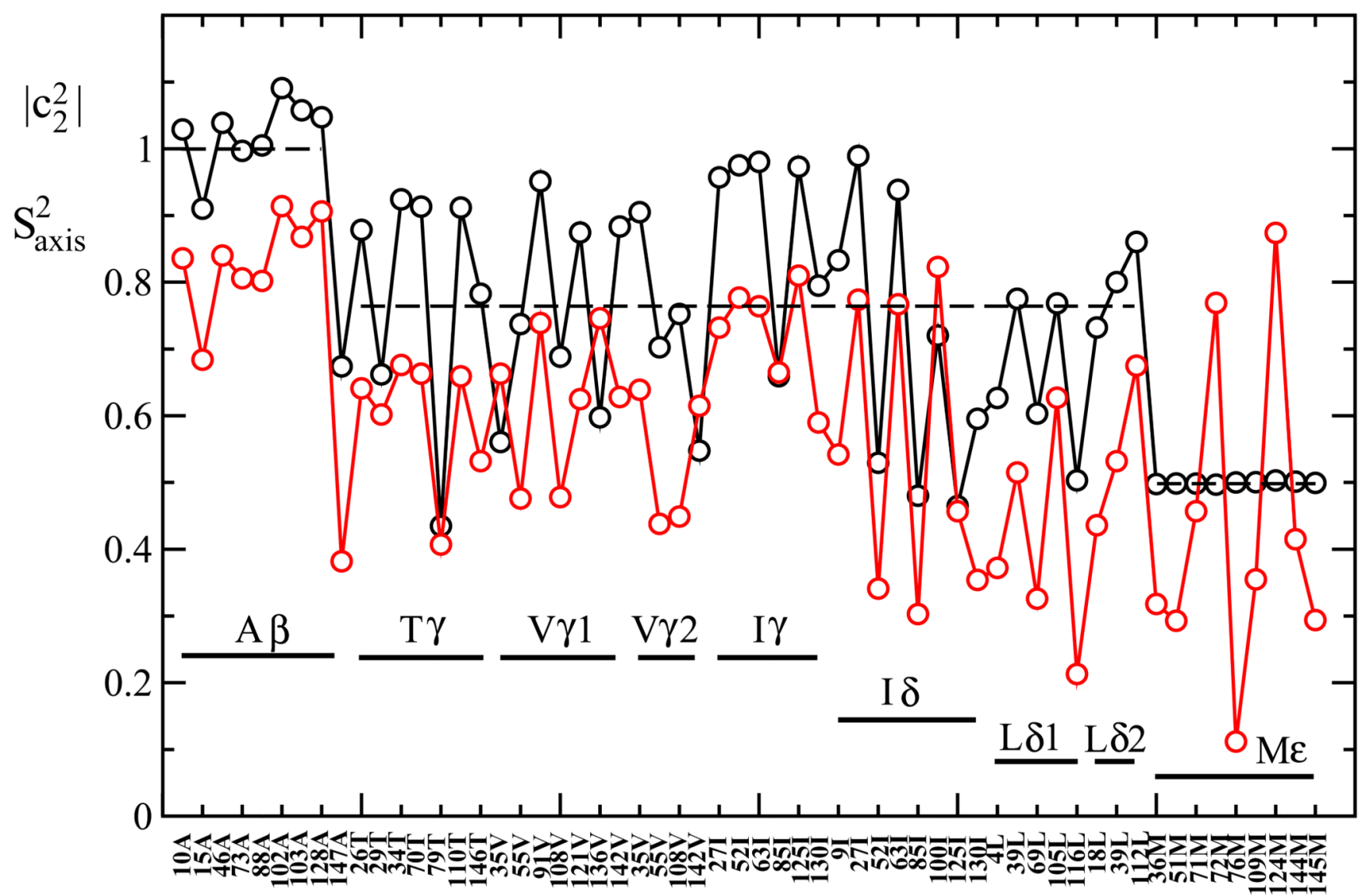

Figure 7.

$\mid c_{2}^{2}$ from Figure 2c (black circles) and the corresponding $S_{\text {axis }}{ }^{2}$ value from ref 20 (red circles) as a function of methyl type, at $295 \mathrm{~K}$. 

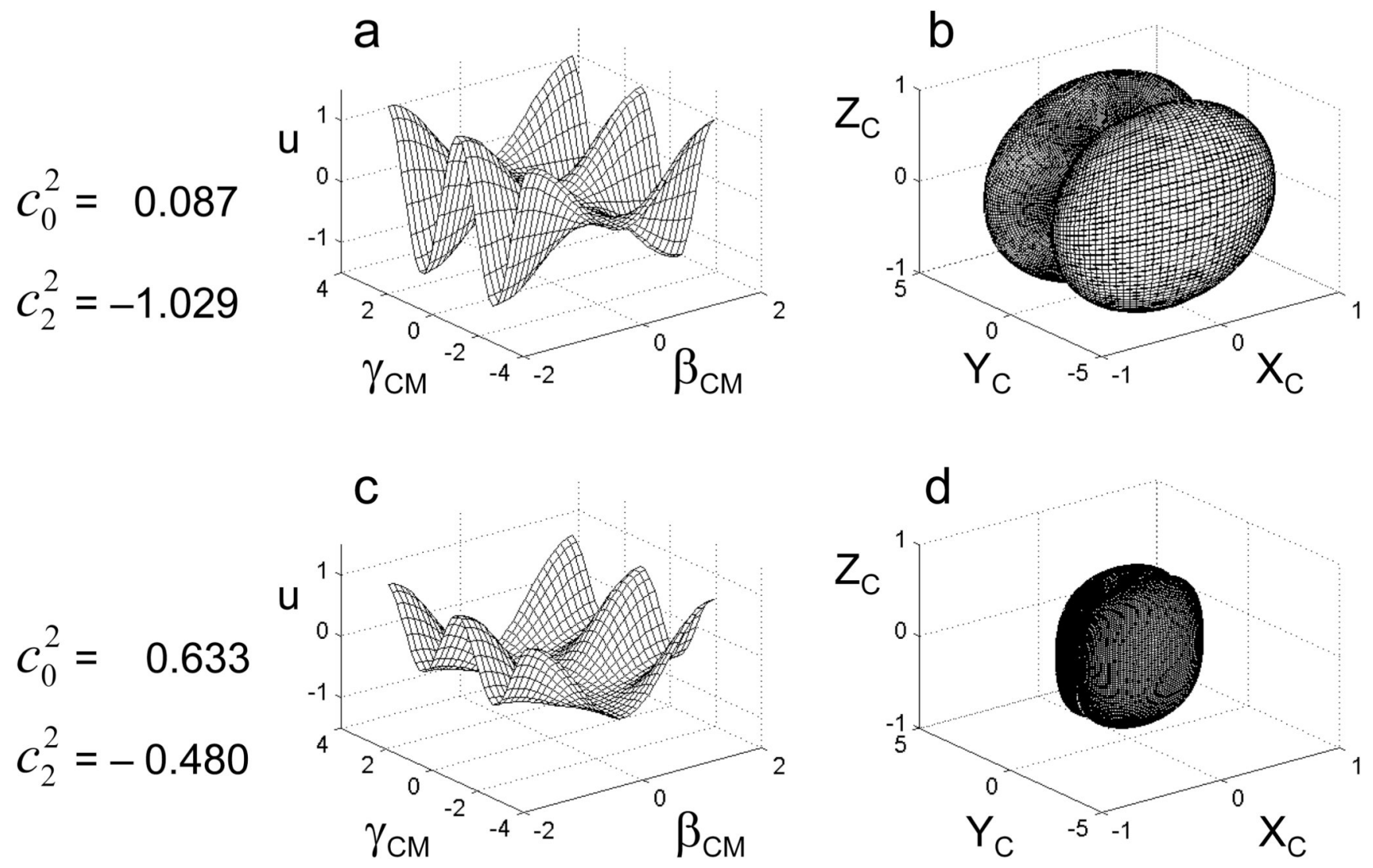

Figure 8.

(a) The potential $u=-0.087 \times\left(1.5 \cos ^{2} \beta_{\mathrm{CM}}-1 / 2\right)+1.089 \times(3 / 2)^{1 / 2} \sin ^{2} \beta_{\mathrm{CM}} \cos 2 \gamma_{\mathrm{CM}}$ as a function of $\beta_{\mathrm{CM}}$ and $\gamma_{\mathrm{CM}}$ given in radians. The potential coefficients are the best-fit values obtained by fitting with SRLS the experimental data of methyl group A10 of $\mathrm{Ca}^{2+}-\mathrm{CaM}^{*}$ smMLCKp, as desctibed in the text. (b) The relative probability $P_{\text {rel }}$ of the $\mathrm{C}-{ }^{13} \mathrm{CDH}_{2}$ axis having an orientation in the infinitesimal range $\beta_{\mathrm{CM}} \pm \Delta \beta_{\mathrm{CM}}$ and $\gamma_{\mathrm{CM}} \pm$ $\Delta \gamma_{\mathrm{CM}}$, for any $\alpha$, given by $\exp \left[0.087 \times\left(3 / 2 \cos ^{2} \beta_{\mathrm{CM}}-1 / 2\right)-1089 \times(3 / 2)^{1 / 2} \sin ^{2}-\right.$ $\left.\beta_{\mathrm{CM}} \cos 2 \gamma_{\mathrm{CM}}\right] \sin \beta_{\mathrm{CM}} \Delta \beta_{\mathrm{CM}} \Delta \gamma_{\mathrm{CM}}$, as a function of the spherical coordinates $\left(\beta_{\mathrm{CM}}, \gamma_{\mathrm{CM}}\right)$. In this context, the uniaxial local director is defined to lie along the equilibrium $\mathrm{C}-{ }^{13} \mathrm{CDH}_{2}$ orientation. (c) The potential $u=-0.633 \times\left(3 / 2 \cos ^{2} \beta_{\mathrm{CM}}-1 / 2\right)+0.480 \times$ $(3 / 2)^{1 / 2} \sin ^{2} \beta_{\mathrm{CM}} \cos 2 \gamma_{\mathrm{CM}}$ as a function of $\beta_{\mathrm{CM}}$ and $\gamma_{\mathrm{CM}}$ given in radians. The potential coefficients are the best-fit values obtained by fitting with SRLS the experimental data of methyl group I $\delta 85$ of $\mathrm{Ca}^{2+}-\mathrm{CaM}^{*} \mathrm{smMLCKp}$ (see text for details). (d) The relative probability of the $\mathrm{C}-{ }^{13} \mathrm{CDH}_{2}$ axis having an orientation in the infinitesimal range $\beta_{\mathrm{CM}} \pm$ $\Delta \beta_{\mathrm{CM}}$ and $\gamma_{\mathrm{CM}} \pm \Delta \gamma_{\mathrm{CM}}$, for any $\alpha$, given by $\exp \left[0.633 \times\left(3 / 2 \cos ^{2} \beta_{\mathrm{CM}}-1 / 2\right)-0.480 \times\right.$ $\left.(3 / 2)^{1 / 2} \sin ^{2} \beta_{\mathrm{CM}} \cos 2 \gamma_{\mathrm{CM}}\right] \sin \beta_{\mathrm{CM}} \Delta \beta_{\mathrm{CM}} \Delta \gamma_{\mathrm{CM}}$, as a function of the spherical coordinates $\left(\beta_{\mathrm{CM}}, \gamma_{\mathrm{CM}}\right)$. 


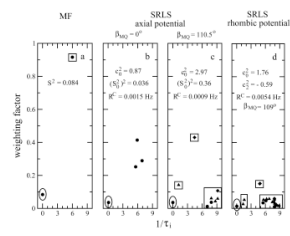

Figure 9.

SRLS eigenmodes (ordinate) and corresponding eigenvalues (abscissa) of $C_{00}(t)$ (circles), $C_{11}(t)$ (triangles) and $C_{22}(t)$ (diamonds). The unitless eigenmode, $1 / \tau_{\mathrm{i}}$, is given in units of the local motional rate, $R^{\mathrm{L}}$. The unitless parameter, $R^{\mathrm{C}}$, represents the time scale separation, $\tau / \tau_{\mathrm{m}}$. The parameters within the panels (except for the $S_{0}^{2}$ values which were calculated from corresponding $c_{0}^{2}$ values) are best-fit values obtained by analyzing with SRLS-based fitting (Figures 8b-d) or MF-based fitting (Figure 8a) the experimental ${ }^{2} \mathrm{H} R_{1}$ and $R_{2}$ data ${ }^{20}$ of methyl group A10 of $\mathrm{Ca}^{2+}-\mathrm{CaM}^{*}$ smMLCKp, acquired at 14.1 and $17.6 \mathrm{~T}$, and $295 \mathrm{~K}$. The parameters on the top were fixed in the respective data fitting calculation. The various panels correspond to different parameter combinations used. The boxes mark clusters of eigenvalues comparable in magnitude. 


\section{Table 1}

Average $c_{0}^{2}$ and $c_{2}^{2}$ values of the methyl groups of $\mathrm{Ca}^{2+}-\mathrm{CaM}^{*} \mathrm{smMLCKp}$ as a function of temperature (rows $1-4)$. Average $c_{0}^{2}$ and $c_{2}^{2}$ values for alanines (A), methionines (M), and all the other methyl groups at $295 \mathrm{~K}$ (rows 5-7).

\begin{tabular}{llccc}
\hline & $\mathbf{T}, \mathbf{K}$ & $\left\langle c_{0}^{2}\right\rangle$ & $\left\langle c_{2}^{2}\right\rangle$ & $\left|\left\langle c_{2}^{2}\right\rangle\right|\left\langle c_{0}^{2}\right\rangle \mid$ \\
1 & 288 & 0.919 & -0.676 & 0.74 \\
2 & 295 & 0.471 & -0.752 & 1.60 \\
3 & 308 & 0.394 & -0.735 & 1.87 \\
4 & 320 & 0.792 & -0.627 & 0.79 \\
5 & $\mathrm{~A}(295)$ & 0.216 & -0.983 & 4.55 \\
6 & Other(295) & 0.489 & -0.757 & 1.55 \\
7 & $\mathrm{M}(295)$ & 0.645 & -0.500 & 0.78 \\
\hline
\end{tabular}




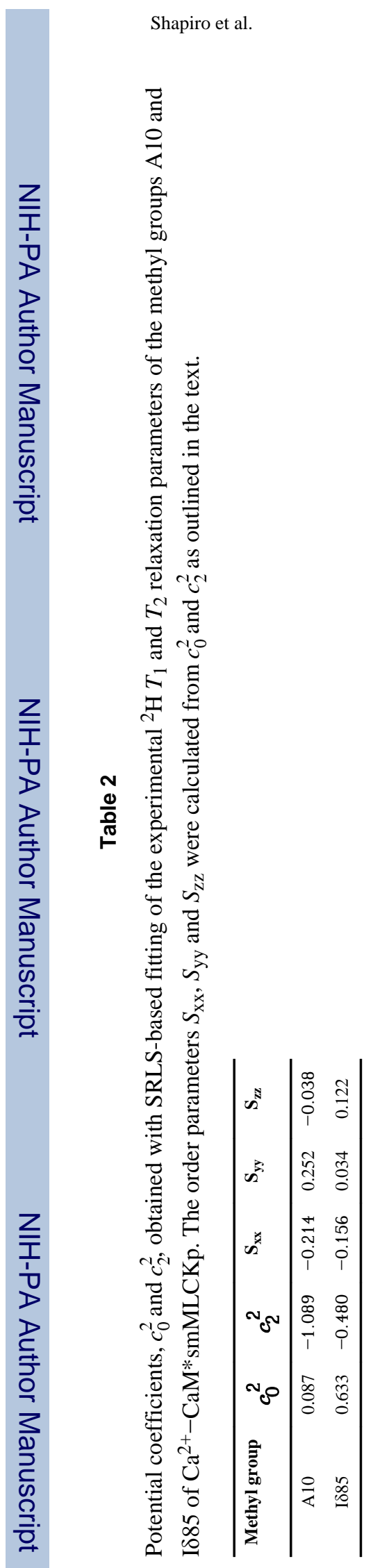

J Phys Chem B. Author manuscript; available in PMC 2012 January 20. 


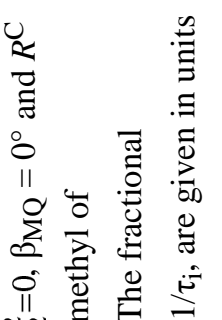

"10

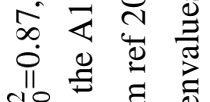

लำ

उ

豞 离

营 N

गु है 3 क

ड

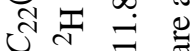

ป II

ส

Є

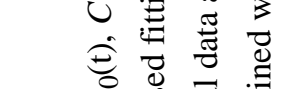

$m$

듕 छ

응 की एँ

佘氙 寻

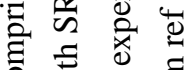

0 范 。

$\therefore$ E

ث.

仓ิ

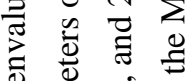

ब. है

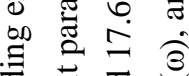

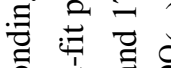

ㅎํㅇ ส व

它亣

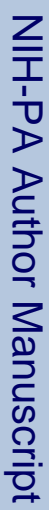

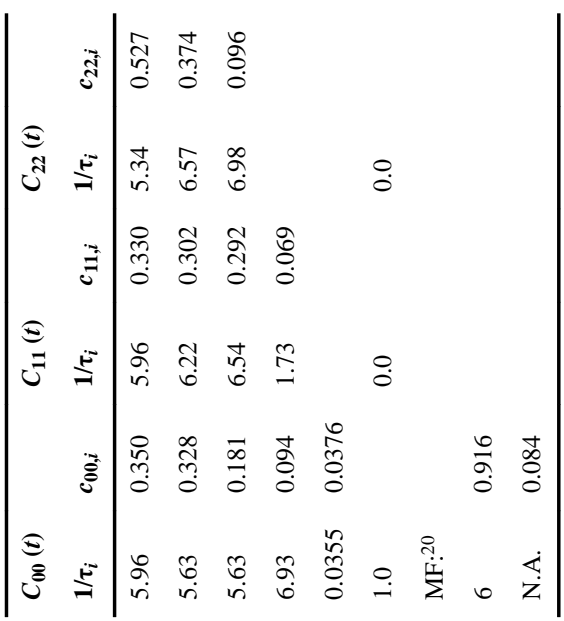




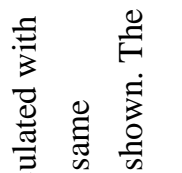

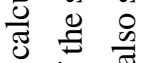

ड़

뉴 (3)

चี

(5)

$\exists$ is n

डิ

\&

苛

氖

क

:

ซ

究

氙 UI

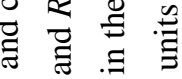

$\therefore$ in.$\Xi$

¿

i

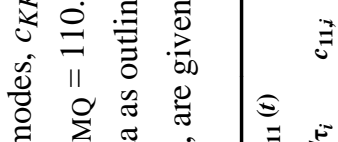

$=\frac{3}{3}$

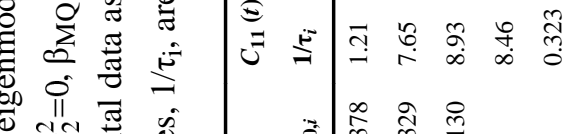

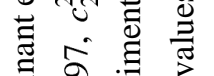




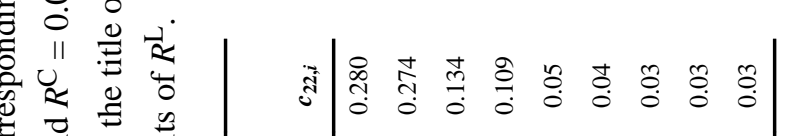

